Mathematical Modelling and Analysis

Volume 22 Number 1, January 2017, 37-51

https://doi.org/10.3846/13926292.2017.1263244

(c) Vilnius Gediminas Technical University, 2017
Publisher: Taylor\&Francis and VGTU

http://www.tandfonline.com/TMMA

ISSN: $1392-6292$

eISSN: 1648-3510

\title{
On the Asymptotic Behavior of Eigenvalues and Eigenfunctions of the Robin Problem with Large Parameter
}

\author{
Alexey V. Filinovskiy ${ }^{a, b}$ \\ ${ }^{a}$ Department of High Mathematics, Faculty of Fundamental Sciences, \\ Bauman Moscow State Technical University \\ 2nd Baumanskaya st. 5, 105005 Moscow, Russia \\ ${ }^{b}$ Department of Differential Equations, Faculty of Mechanics and \\ Mathematics, Lomonosov Moscow State University \\ GSP-1, Leninskie Gory 1, 119991 Moscow, Russia \\ E-mail(corresp.): flnv@yandex.ru \\ E-mail: flnv@bmstu.ru
}

Received April 12, 2016; revised November 15, 2016; published online January 5, 2017

\begin{abstract}
We consider the eigenvalue problem with Robin boundary condition $\Delta u+\lambda u=0$ in $\Omega, \partial u / \partial \nu+\alpha u=0$ on $\partial \Omega$, where $\Omega \subset \mathbb{R}^{n}, n \geq 2$ is a bounded domain with a smooth boundary, $\nu$ is the outward unit normal, $\alpha$ is a real parameter. We obtain two terms of the asymptotic expansion of simple eigenvalues of this problem for $\alpha \rightarrow+\infty$. We also prove an estimate to the difference between Robin and Dirichlet eigenfunctions.
\end{abstract}

Keywords: Laplace operator, Robin boundary condition, eigenvalues and eigenfunctions, large parameter, asymptotic behavior.

AMS Subject Classification: 35P15; 35J05.

\section{Introduction}

Let $\Omega \subset \mathbb{R}^{n}, n \geq 2$ be a bounded domain with boundary $\Gamma$ of class $C^{3}$. Consider the eigenvalue problem

$$
\begin{aligned}
& \Delta u+\lambda u=0 \quad \text { in } \Omega, \\
& \frac{\partial u}{\partial \nu}+\alpha u=0 \quad \text { on } \Gamma,
\end{aligned}
$$

where $\nu$ is the outward unit normal vector to $\Gamma, \alpha$ is a real parameter. By $\left\{\lambda_{k}(\alpha)\right\}_{k=1}^{\infty}$ we denote the sequence of eigenvalues of the problem (1.1), (1.2) 
enumerated as

$$
\lambda_{k}(\alpha)=\sup _{v_{1}, \ldots, v_{k-1} \in L_{2}(\Omega)} \inf _{\substack{v \in H^{1}(\Omega),\left(v, v_{j}\right)_{L_{2}(\Omega)=0}=0 \\ j=1, \ldots, k-1}} \frac{\int_{\Omega}|\nabla v|^{2} d x+\alpha \int_{\Gamma} v^{2} d s}{\int_{\Omega} v^{2} d x}
$$

for $k=1,2, \ldots$ and by $\left\{\lambda_{k}^{D}\right\}_{k=1}^{\infty}$ denote the sequence of eigenvalues of Dirichlet problem

$$
\Delta u+\lambda u=0 \text { in } \Omega, \quad u=0 \text { on } \Gamma,
$$

enumerated respectively

$$
\lambda_{k}^{D}=\sup _{v_{1}, \ldots, v_{k-1} \in L_{2}(\Omega)} \inf _{\substack{v \in H^{1}(\Omega) \\\left(v, v_{j}\right)_{L_{2}(\Omega)}=0 \\ j=1, \ldots, k-1}} \frac{\int_{\Omega}|\nabla v|^{2} d x}{\int_{\Omega} v^{2} d x}
$$

for $k=1,2, \ldots$.

By $\left\{u_{k, \alpha}(x)\right\}_{k=1}^{\infty}$ and $\left\{u_{k}^{D}(x)\right\}_{k=1}^{\infty}$ we denote the corresponding sequences of normalized in $L_{2}(\Omega)$ Robin and Dirichlet eigenfunctions (all considered functional and abstract spaces will be real spaces). It is known that the eigenvalues $\lambda_{1}(\alpha)$ and $\lambda_{1}^{D}>0$ are simple and $\lim _{k \rightarrow \infty} \lambda_{k}(\alpha)=\lim _{k \rightarrow \infty} \lambda_{k}^{D}=+\infty$. It follows from (1.3), (1.5) that $\lambda_{k}(\alpha) \leq \lambda_{k}^{D}$. As it was noted in ( [3], Chapter 6, Section 2, Subsection 1) for $n=2$ in the case of a smooth boundary $\Gamma$ we have $\lim _{\alpha \rightarrow+\infty} \lambda_{k}(\alpha)=\lambda_{k}^{D}, k=1,2, \ldots$ Later in [19] for $n=2$ the following two-side estimates for the first eigenvalue $\lambda_{1}(\alpha)$ were obtained

$$
\lambda_{1}^{D}\left(1+\frac{\lambda_{1}^{D}}{\alpha q_{1}}\right)^{-1} \leq \lambda_{1}(\alpha) \leq \lambda_{1}^{D}\left(1+\frac{4 \pi}{\alpha|\Gamma|}\right)^{-1}, \quad \alpha>0,
$$

where $q_{1}$ is the first eigenvalue of Steklov problem

$$
\begin{aligned}
\Delta^{2} u & =0 & & \text { in } \Omega, \\
u=0, & \Delta u-q \frac{\partial u}{\partial \nu}=0 & & \text { on } \Gamma .
\end{aligned}
$$

In $[7,8]$, the estimates for all eigenvalues were proved

$$
\lambda_{k}^{D}-C_{1} \frac{\left(\lambda_{k}^{D}\right)^{2}}{\sqrt{\alpha}} \leq \lambda_{k}(\alpha) \leq \lambda_{k}^{D}, \quad \alpha>\alpha_{1}>0
$$

where constants $C_{1}$ and $\alpha_{1}$ are independent on $k$. In [9] the estimates (1.6) were sharpened:

$$
\lambda_{k}^{D}-C_{2} \frac{\left(\lambda_{k}^{D}\right)^{2}}{\alpha} \leq \lambda_{k}(\alpha) \leq \lambda_{k}^{D}, \quad \alpha>\alpha_{1}>0 .
$$

The case $\alpha<0$ has recently attracted attention in connection with diffusion models [12]. It was shown in [12] that for piecewise $C^{1}$ class boundary

$$
\liminf _{\alpha \rightarrow-\infty} \frac{\lambda_{1}(\alpha)}{-\alpha^{2}} \geq 1
$$


and in particular when $\Omega \subset \mathbb{R}^{2}$ is a triangle with inner half-angles $\alpha_{1}, \alpha_{2}, \alpha_{3}$ the authors have proved that

$$
\lim _{\alpha \rightarrow-\infty} \frac{\lambda_{1}(\alpha)}{-\alpha^{2}}=\max _{1 \leq j \leq 3} \operatorname{cosec}^{2} \alpha_{j}>1 .
$$

In [14] for $C^{1}$ class boundaries the following equality was proved

$$
\lim _{\alpha \rightarrow-\infty} \frac{\lambda_{1}(\alpha)}{-\alpha^{2}}=1
$$

These results indicate that the asymptotic behavior of $\lambda_{1}(\alpha)$ for $\alpha \rightarrow-\infty$ is strongly affected by the smoothness of the boundary and $C^{1}$ class is optimal for the equality (1.8). In [4] it was proved that for $C^{1}$ boundaries for all $k=1,2, \ldots$ we have

$$
\lim _{\alpha \rightarrow-\infty} \frac{\lambda_{k}(\alpha)}{-\alpha^{2}}=1
$$

The second term of asymptotics of $\lambda_{k}(\alpha) /\left(-\alpha^{2}\right)$ was found in [5] for $n=2$ with $\Gamma \in C^{4}$ and later in [17] for $n \geq 2$ with $\Gamma \in C^{3}$ :

$$
\frac{\lambda_{k}(\alpha)}{-\alpha^{2}}=1-(n-1) H_{\max } \alpha^{-1}+O\left(|\alpha|^{-4 / 3}\right), \quad \alpha \rightarrow-\infty
$$

with $H_{\max }=\max _{x \in \Gamma} H(x)$ where $H(x)$ is the mean curvature of the surface $\Gamma$ oriented by inner normal at the point $x$. It was proved in [17], that if, additionally, $\Gamma \in C^{4}$, then the reminder estimate $O\left(|\alpha|^{-4 / 3}\right)$ can be replaced by $O\left(|\alpha|^{-3 / 2}\right)$. Let us note the result of [7], where the lower estimate for the derivative of the first eigenvalue $\lambda_{1}^{\prime}(\alpha)$ were obtained:

$$
\liminf _{\alpha \rightarrow-\infty} \frac{\lambda_{1}^{\prime}(\alpha)}{-\alpha} \geq 1
$$

\section{Main results}

By $m(\lambda)$ we denote the multiplicity of eigenvalue $\lambda$.

Theorem 1. For any $k=1,2, \ldots$ there exists the number $\alpha_{k} \in \mathbb{R}$ such that for $\alpha>\alpha_{k}$ we have

$$
m\left(\lambda_{k}(\alpha)\right) \leq m\left(\lambda_{k}^{D}\right) .
$$

Theorem 2. Let $m\left(\lambda_{k}^{D}\right)=1$. Then there exists the number $\alpha_{k} \in \mathbb{R}$ such that for all $\alpha>\alpha_{k}$ the eigenvalue $\lambda_{k}(\alpha)$ is a differentiable function and

$$
\lambda_{k}^{\prime}(\alpha)=\frac{\int_{\Gamma} u_{k, \alpha}^{2} d s}{\int_{\Omega} u_{k, \alpha}^{2} d x}>0 .
$$

Theorem 3. Let $m\left(\lambda_{k}^{D}\right)=1$. Then the eigenvalue $\lambda_{k}(\alpha)$ obeys an asymptotic expansion

$$
\lambda_{k}(\alpha)=\lambda_{k}^{D}-\frac{\int_{\Gamma}\left(\frac{\partial u_{k}^{D}}{\partial \nu}\right)^{2} d s}{\int_{\Omega}\left(u_{k}^{D}\right)^{2} d x} \alpha^{-1}+o\left(\alpha^{-1}\right), \quad \alpha \rightarrow+\infty .
$$


We can see that

$$
\int_{\Gamma}\left(\frac{\partial u_{k}^{D}}{\partial \nu}\right)^{2} d s>0 .
$$

If not, the eigenfunction $u_{k}^{D}$ is a solution of the Cauchy problem

$$
\Delta u_{k}^{D}+\lambda_{k}^{D} u_{k}^{D}=0 \text { in } \Omega, \quad u_{k}^{D}=\partial u_{k}^{D} / \partial \nu=0 \text { on } \Gamma .
$$

So, $u_{k}^{D} \equiv 0$ ( [11], Chapter 1, Theorem 1.46). This is impossible. The relations $(2.2),(2.3)$ show that the first power of $\alpha$ in the denominator of (1.7) cannot be replaced by $\alpha^{1+\delta}$ with any $\delta>0$.

Remark. For the first eigenvalue the expansion (2.2) was obtained in [6].

The next theorem establishes the rate of convergence of the eigenfunction $u_{k, \alpha}$ of the Robin problem (1.1), (1.2) to the eigenfunction $u_{k}^{D}$ of the "limit" Dirichlet problem (1.4) at $\alpha \rightarrow+\infty$. For any $\alpha \in \mathbb{R}$ we suppose $\int_{\Omega} u_{k, \alpha} u_{k}^{D} d x \geq$ 0 .

Theorem 4. Let $m\left(\lambda_{k}^{D}\right)=1$. Then there exists the number $\alpha_{k} \in \mathbb{R}$ such that for all $\alpha>\alpha_{k}$ we have $m\left(\lambda_{k}(\alpha)\right)=1$ and

$$
\left\|u_{k, \alpha}-u_{k}^{D}\right\|_{H^{2}(\Omega)} \leq M_{k} / \alpha
$$

where the constant $M_{k}$ is independent of $\alpha$.

Let us notify the difference of asymptotic behavior of eigenvalues in our case $\alpha>0$ (repulsive Robin condition) with the case $\alpha<0$ (attractive Robin condition). Here for smooth boundary the global properties of the eigenfunctions (2.4) determine the second term of the asymptotics (2.2), while in the attractive case the second term of the asymptotics (1.9) is determined by a local behavior of the boundary around the points of the maximum mean curvature.

\section{Perturbation of eigenvalues}

We use the following inequality ( [16], Lemma 51.1).

Theorem 5. Let $A$ and $B$ be two linear self-adjoint, compact and positive operators on a separable Hilbert space $H$. Let $\mu_{k}^{A}$ and $\mu_{k}^{B}$ be their eigenvalues, enumerated in the decreasing order according to their multiplicity. Then

$$
\left|\mu_{k}^{A}-\mu_{k}^{B}\right| \leq\|A-B\| .
$$

For $h(x) \in L_{2}(\Omega)$ consider the weak solution $u(x) \in H^{1}(\Omega)$ of the Robin boundary value problem

$$
\begin{array}{ll}
-\Delta u+u=h & \text { in } \Omega, \\
\frac{\partial u}{\partial \nu}+\alpha u=0 & \text { on } \Gamma, \quad \alpha>0 .
\end{array}
$$

In domain with $C^{2}$ class boundary surface we have $u \in H^{2}(\Omega)$ ( [15], Chapter 4, Section 2, Theorem 4). For our proofs we need an elliptic estimate ( [9], Theorem 3) to solution of the problem (3.2), (3.3) at large values of $\alpha$. 
Theorem 6. The solution of the problem (3.2), (3.3) satisfies the estimate

$$
\|u\|_{H^{2}(\Omega)} \leq C_{3}\|h\|_{L_{2}(\Omega)}, \quad \alpha>\alpha_{1}>0
$$

with the constant $C_{3}$ is independent of $\alpha$.

Remark. Let us note that the estimates of type (3.4) to solution of the problem (3.2), (3.3) were previously obtained for fixed $\alpha$ (see, for example, [15]). But we need the estimate (3.4) valid with one constant $C_{3}$ for $\alpha \rightarrow+\infty$.

For Robin problem (3.2), (3.3) we define the linear operator $A_{\alpha}$ by

$$
A_{\alpha}: L_{2}(\Omega) \rightarrow H^{1}(\Omega) \subset L_{2}(\Omega) \text { for } h \mapsto u .
$$

The operator $A_{\alpha}$ is a self-adjoint positive compact operator in $L_{2}(\Omega)$ ( [8], Section 4) and $\left\|A_{\alpha}\right\|=1 /\left(\lambda_{1}(\alpha)+1\right)<1$. Now,

$$
\left(\lambda_{k}(\alpha)+1\right) A_{\alpha} u_{k, \alpha}=u_{k, \alpha},
$$

thence

$$
\mu_{k}(\alpha)=1 /\left(\lambda_{k}(\alpha)+1\right)
$$

are the eigenvalues of $A_{\alpha}$ with the eigenfunctions $u_{k, \alpha} \in H^{1}(\Omega)$.

Let $w=\left(A_{\tilde{\alpha}}-A_{\alpha}\right) h=\tilde{u}-u$. The function $w$ is a solution of the boundary value problem

$$
\begin{aligned}
& -\Delta w+w=0 \quad \text { in } \Omega, \\
& \frac{\partial w}{\partial \nu}+\alpha w=(\tilde{\alpha}-\alpha) \tilde{u} \quad \text { on } \Gamma \text {. }
\end{aligned}
$$

It follows from (3.6), (3.7) that

$$
\int_{\Omega}\left(|\nabla w|^{2}+w^{2}\right) d x+\alpha \int_{\Gamma} w^{2} d s=(\alpha-\tilde{\alpha}) \int_{\Gamma} w \tilde{u} d s .
$$

Thence, for $\alpha>\alpha_{1}>0$

$$
\int_{\Omega}\left(|\nabla w|^{2}+w^{2}\right) d x+\frac{\alpha}{2} \int_{\Gamma} w^{2} d s \leq \frac{(\tilde{\alpha}-\alpha)^{2}}{2 \alpha} \int_{\Gamma} \tilde{u}^{2} d s \leq C_{4}(\tilde{\alpha}-\alpha)^{2}\|\tilde{u}\|_{H^{1}(\Omega)}^{2}
$$

and

$$
\|w\|_{H^{1}(\Omega)} \leq C_{5}|\tilde{\alpha}-\alpha|\|\tilde{u}\|_{H^{1}(\Omega)} .
$$

Consequentially,

$$
\left\|\left(A_{\tilde{\alpha}}-A_{\alpha}\right) h\right\|_{L_{2}(\Omega)} \leq C_{5}|\tilde{\alpha}-\alpha|\|\tilde{u}\|_{H^{1}(\Omega)} .
$$

Now, applying the estimate (3.4) for $h \in L_{2}(\Omega)$ we have the inequalities

$$
\begin{aligned}
& \left\|\left(A_{\tilde{\alpha}}-A_{\alpha}\right) h\right\|_{L_{2}(\Omega)} \leq C_{5}|\tilde{\alpha}-\alpha|\|h\|_{L_{2}(\Omega)}, \\
& \left\|A_{\tilde{\alpha}}-A_{\alpha}\right\| \leq C_{5}|\tilde{\alpha}-\alpha|, \quad \alpha, \tilde{\alpha}>\alpha_{1} .
\end{aligned}
$$


Then by the inequalities (3.1), (3.8) we get

$$
\left|\mu_{k}(\tilde{\alpha})-\mu_{k}(\alpha)\right| \leq C_{5}|\tilde{\alpha}-\alpha| .
$$

Therefore,

$$
\left|\lambda_{k}(\tilde{\alpha})-\lambda_{k}(\alpha)\right| \leq C_{5}\left(\lambda_{k}(\tilde{\alpha})+1\right)\left(\lambda_{k}(\alpha)+1\right)|\tilde{\alpha}-\alpha| .
$$

Furthermore, consider the Dirichlet problem

$$
\begin{aligned}
-\Delta v+v & =h & & \text { in } \Omega, \\
v & =0 & & \text { on } \Gamma .
\end{aligned}
$$

We define the linear operator $A^{D}$ by

$$
A^{D}: L_{2}(\Omega) \rightarrow \stackrel{o}{H^{1}}(\Omega) \subset L_{2}(\Omega), \text { for } h \mapsto v .
$$

The operator $A^{D}$ is a self-adjoint positive compact operator in $L_{2}(\Omega)$ ( $[8]$, Section 4) and $\left\|A^{D}\right\|=1 /\left(\lambda_{1}^{D}+1\right)<1$. We have

$$
\left(\lambda_{k}^{D}+1\right) A^{D} u_{k}^{D}=u_{k}^{D},
$$

therefore

$$
\mu_{k}^{D}=1 /\left(\lambda_{k}^{D}+1\right), \quad k=1,2, \ldots
$$

are the eigenvalues of $A^{D}$ with the eigenfunctions $u_{k}^{D} \in \stackrel{o}{H^{1}}(\Omega)$.

Let $w=\left(A^{D}-A_{\alpha}\right) h=v-u$. By (3.2), (3.3), (3.10), (3.11) the function $w$ is a solution of the boundary value problem

$$
-\Delta w+w=0 \quad \text { in } \Omega, \quad w=\frac{1}{\alpha} \frac{\partial u}{\partial \nu} \quad \text { on } \Gamma
$$

where $u=A_{\alpha} h \in H^{2}(\Omega)$ ( [15], Chapter 4, Section 2, Theorem 4). Due to elliptic regularity ( $[2]$, Par. 11.1d) we have the estimate

$$
\|w\|_{H^{1}(\Omega)} \leq \frac{C_{6}}{\alpha}\left\|\frac{\partial u}{\partial \nu}\right\|_{H^{1 / 2}(\Gamma)}
$$

and consequently by the embedding theorem for Sobolev spaces ( $[2]$, Theorem $5.1 .7)$

$$
\left\|\left(A^{D}-A_{\alpha}\right) h\right\|_{L_{2}(\Omega)} \leq \frac{C_{7}}{\alpha}\|u\|_{H^{2}(\Omega)} .
$$

Now, applying the estimate (3.4) for $h \in L_{2}(\Omega)$, we have the following inequalities

$$
\left\|\left(A^{D}-A_{\alpha}\right) h\right\|_{L_{2}(\Omega)} \leq \frac{C_{3} C_{7}}{\alpha}\|h\|_{L_{2}(\Omega)},\left\|A^{D}-A_{\alpha}\right\| \leq \frac{C_{3} C_{7}}{\alpha}, \quad \alpha>\alpha_{1} .
$$

Then by the equalities (3.5), (3.12) and the inequalities (3.1), (3.13) we get the estimate

$$
\left|\mu_{k}^{D}-\mu_{k}(\alpha)\right| \leq C_{3} C_{7} / \alpha
$$


Therefore,

$$
\left|\lambda_{k}^{D}-\lambda_{k}(\alpha)\right| \leq \frac{C_{3} C_{7}}{\alpha}\left(\lambda_{k}^{D}+1\right)\left(\lambda_{k}(\alpha)+1\right)
$$

and taking into account the inequalities $\lambda_{k}(\alpha) \leq \lambda_{k}^{D}$, we obtain

$$
0 \leq \lambda_{k}^{D}-\lambda_{k}(\alpha) \leq \frac{C_{3} C_{7}}{\alpha}\left(\lambda_{k}^{D}+1\right)^{2} \leq \frac{C_{2}}{\alpha}\left(\lambda_{k}^{D}\right)^{2} .
$$

Proof of Theorem 1. Let $m=m\left(\lambda_{k}^{D}\right)$ and

$$
\lambda_{k-1}^{D}<\lambda_{k}^{D}=\lambda_{k+1}^{D}=\cdots=\lambda_{k+m-1}^{D}<\lambda_{k+m}^{D} .
$$

Now we consider the eigenvalues $\lambda_{j}(\alpha), j=k, k+1, \ldots, k+m-1$ as functions of the variable $\alpha$. It follows from (3.14) and (3.15) that for any $\varepsilon>0$ there exists $\alpha_{k} \in \mathbb{R}$ such that for all $\alpha>\alpha_{k}$ the following inequalities hold

$$
\begin{aligned}
& \lambda_{j}(\alpha)<\lambda_{k-1}^{D}+\varepsilon, \quad j=1,2, \ldots, k-1, \\
& \lambda_{j}(\alpha)>\lambda_{k+m}^{D}-\varepsilon, \quad j=k+m, k+m+1, \ldots .
\end{aligned}
$$

Let $\varepsilon=\min \left\{\left|\lambda_{k}^{D}-\lambda_{k-1}^{D}\right|,\left|\lambda_{k+m}^{D}-\lambda_{k}^{D}\right|\right\} / 2$. Then by (3.16), (3.17) for $\alpha>\alpha_{k}$ the interval $\left(\lambda_{k}^{D}-\varepsilon, \lambda_{k}^{D}+\varepsilon\right)$ contains only the eigenvalues $\lambda_{j}(\alpha), j=k, \ldots, k+m-1$. The other eigenvalues of the problem (1.1), (1.2) are located outside of this interval. Thus, for any $\alpha>\alpha_{k}$ only the functions $\lambda_{j}(\alpha), j=k, \ldots, k+m-1$ can be equal and a number of such functions does not exceeds $m$. Theorem 1 is proved.

\section{Perturbation of eigenvectors: abstract model}

Consider a real Hilbert space $H$ with the scalar product $(\cdot, \cdot)$ and the norm $\|\cdot\|$. Let $e$ and $g$ be non-zero elements in $H$.

Definition 1. The non-negative number

$$
\delta(e, g)=\sqrt{1-(e, g)^{2} /\|e\|^{2}\|g\|^{2}}
$$

is called a deviation between $e$ and $g$.

It follows from (4.1) that $0 \leq \delta(e, g) \leq 1, \delta(e, g)=\delta(g, e), \delta(e, g) \leq \delta(e, h)+$ $\delta(h, g)$ and the linear spans of $e$ and $g$ coincides if and only if $\delta(e, g)=0$. Therefore, the deviation is a metric on the set of one-dimensional linear subspaces of $H$.

Lemma 1. Let $(e, g) \geq 0$. Then the following estimate holds:

Proof of Lemma 1.

$$
\|e /\| e\|-g /\| g\|\| \leq \sqrt{2} \delta(e, g) .
$$

$$
\begin{aligned}
\|e /\| e\|-g /\| g\|\|^{2} & =2(1-(e, g) /\|e\|\|g\|) \\
& =2 \delta^{2}(e, g) /(1+(e, g) /\|e\|\|g\|) \leq 2 \delta^{2}(e, g) .
\end{aligned}
$$


Let $A$ be a linear compact self-adjoint positive operator in $H$. Let $\left\{\mu_{j}\right\}_{j=1}^{\infty}$ be a sequence of its eigenvalues and $\left\{e_{j}\right\}_{j=1}^{\infty}$ be an orthonormal basis of corresponding eigenvectors.

Definition 2. The number $\varrho_{k}^{A}=\inf _{j \neq k}\left|\mu_{j}-\mu_{k}\right|$ is called an isolation distance of the eigenvalue $\mu_{k}$.

The following Lemma 2 generalizes Lemma 1 from [10].

Lemma 2. Let $z \in H$ and $z \neq 0$. Then for any $k=1,2, \ldots$ we have

$$
\left\|\left(A-\mu_{k} I\right) z\right\| \geq \varrho_{k}^{A} \delta\left(z, e_{k}\right)\|z\| .
$$

Proof of Lemma 2. Let $z=\sum_{j=1}^{\infty} a_{j} e_{j}$. Then

$$
\begin{aligned}
& \left\|\left(A-\mu_{k} I\right) z\right\|^{2}=\left\|\sum_{j=1}^{\infty}\left(\mu_{j}-\mu_{k}\right) a_{j} e_{j}\right\|^{2} \\
& =\sum_{\substack{j=1 \\
j \neq k}}^{\infty}\left(\mu_{j}-\mu_{k}\right)^{2} a_{j}^{2} \geq\left(\varrho_{k}^{A}\right)^{2} \sum_{\substack{j=1 \\
j \neq k}}^{\infty} a_{j}^{2}=\left(\varrho_{k}^{A}\right)^{2}\left(\|z\|^{2}-a_{k}^{2}\right) .
\end{aligned}
$$

It follows from (4.1) that $a_{k}^{2}=\left(1-\delta^{2}\left(z, e_{k}\right)\right)\|z\|^{2}$, so, apply this to (4.2), we obtain $\left\|\left(A-\mu_{k} I\right) z\right\|^{2} \geq\left(\varrho_{k}^{A}\right)^{2} \delta^{2}\left(z, e_{k}\right)\|z\|^{2}$. Lemma 2 is proved.

Let $A$ and $B$ be two linear compact self-adjoint positive operators in $H$ with the sequences of eigenvalues $\left\{\mu_{j}^{A}\right\}_{j=1}^{\infty},\left\{\mu_{j}^{B}\right\}_{j=1}^{\infty}$ and orthonormal bases of eigenvectors $\left\{e_{j}\right\}_{j=1}^{\infty},\left\{g_{j}\right\}_{j=1}^{\infty}$.

Theorem 7. Let for some $k$ holds $\max \left\{\varrho_{k}^{A}, \varrho_{k}^{B}\right\}>0$. Then

$$
\delta\left(e_{k}, g_{k}\right) \leq \frac{2}{\max \left\{\varrho_{k}^{A}, \varrho_{k}^{B}\right\}}\|A-B\| .
$$

Proof of Theorem \%. We have the equality

$$
\left(A-\mu_{k}^{A} I\right)\left(g_{k}-\left(e_{k}, g_{k}\right) e_{k}\right)=(A-B) g_{k}+\left(\mu_{k}^{B}-\mu_{k}^{A}\right) g_{k} .
$$

Since $\left\|e_{k}\right\|=\left\|g_{k}\right\|=1$ we obtain

$$
\delta\left(e_{k}, g_{k}\right)=\sqrt{1-\left(e_{k}, g_{k}\right)^{2}}=\left\|g_{k}-\left(e_{k}, g_{k}\right) e_{k}\right\| .
$$

Now, if $g_{k}=\left(e_{k}, g_{k}\right) e_{k}$, then $\delta\left(e_{k}, g_{k}\right)=0$ and the inequality (4.3) holds. Otherwise, $\left(g_{k}-\left(e_{k}, g_{k}\right) e_{k}, e_{k}\right)=0$ so $\delta\left(g_{k}-\left(e_{k}, g_{k}\right) e_{k}, e_{k}\right)=1$ and by equalities (4.4), (4.5) and Lemma 2 we obtain

$$
\begin{aligned}
\delta\left(e_{k}, g_{k}\right) & \leq \frac{1}{\varrho_{k}^{A}}\left\|\left(A-\mu_{k}^{A} I\right)\left(g_{k}-\left(e_{k}, g_{k}\right) e_{k}\right)\right\| \\
& \leq \frac{1}{\varrho_{k}^{A}}\left(\|A-B\|+\left|\mu_{k}^{A}-\mu_{k}^{B}\right|\right) .
\end{aligned}
$$


Thence, by the Theorem 5

$$
\delta\left(e_{k}, g_{k}\right) \leq \frac{2}{\varrho_{k}^{A}}\|A-B\| .
$$

Interchange operators $A$ and $B$ in (4.6), we have

$$
\delta\left(e_{k}, g_{k}\right) \leq \frac{2}{\varrho_{k}^{B}}\|A-B\| .
$$

Combine estimates (4.6) and (4.7), we finally obtain

$$
\delta\left(e_{k}, g_{k}\right) \leq \frac{2}{\max \left\{\varrho_{k}^{A}, \varrho_{k}^{B}\right\}}\|A-B\| .
$$

This completes the proof of Theorem 7 .

\section{Convergence of eigenfunctions}

For $\tilde{\alpha}, \alpha \in \mathbb{R}$ we suppose $\int_{\Omega} u_{k, \tilde{\alpha}} u_{k, \alpha} d x \geq 0$.

Lemma 3. Let $m\left(\lambda_{k}^{D}\right)=1$. Then there exists the number $\alpha_{k} \in \mathbb{R}$ such that for all $\tilde{\alpha}, \alpha>\alpha_{k}$ we have $m\left(\lambda_{k}(\alpha)\right)=1$ and the following inequality holds

$$
\left\|u_{k, \tilde{\alpha}}-u_{k, \alpha}\right\|_{H^{2}(\Omega)} \leq C_{8}|\tilde{\alpha}-\alpha|
$$

Proof of Lemma 3. It follows from the equality $m\left(\lambda_{k}^{D}\right)=1$ and the Theorem 1 that $\varrho_{k}^{A_{\alpha}}>0, \alpha>\alpha_{k}$ for some $\alpha_{k}$. By the estimates (3.8) and (4.3), applying Theorem 7 for $H=L_{2}(\Omega)$ with $A=A_{\tilde{\alpha}}, B=A_{\alpha}$, we get

$$
\delta\left(u_{k, \tilde{\alpha}}, u_{k, \alpha}\right) \leq \frac{2}{\varrho_{k}^{A_{\alpha}}}\left\|A_{\tilde{\alpha}}-A_{\alpha}\right\| \leq \frac{2 C_{5}}{\varrho_{k}^{A_{\alpha}}}|\tilde{\alpha}-\alpha| .
$$

Thence, it follows from (5.1) and Lemma 1 that

$$
\left\|u_{k, \tilde{\alpha}}-u_{k, \alpha}\right\|_{L_{2}(\Omega)} \leq \frac{2 \sqrt{2} C_{5}}{\varrho_{k}^{A_{\alpha}}}|\tilde{\alpha}-\alpha| .
$$

Now, we have the problems

$$
\begin{aligned}
& \Delta u_{k, \tilde{\alpha}}+\lambda_{k}(\tilde{\alpha}) u_{k, \tilde{\alpha}}=0 \quad \text { in } \Omega, \quad \frac{\partial u_{k, \tilde{\alpha}}}{\partial \nu}+\tilde{\alpha} u_{k, \tilde{\alpha}}=0 \quad \text { on } \Gamma, \\
& \Delta u_{k, \alpha}+\lambda_{k}(\alpha) u_{k, \alpha}=0 \quad \text { in } \Omega, \quad \frac{\partial u_{k, \alpha}}{\partial \nu}+\alpha u_{k, \alpha}=0 \quad \text { on } \Gamma \text {. }
\end{aligned}
$$

By $(5.3)-(5.4)$ the function $w=u_{k, \tilde{\alpha}}-u_{k, \alpha}$ is a solution of the boundary value problem

$$
\begin{aligned}
-\Delta w+w & =\left(\lambda_{k}(\alpha)+1\right) w+\left(\lambda_{k}(\tilde{\alpha})-\lambda_{k}(\alpha)\right) u_{k, \alpha} & & \text { in } \Omega \\
\frac{\partial w}{\partial \nu}+\alpha w & =(\alpha-\tilde{\alpha}) u_{k, \tilde{\alpha}} & & \text { on } \Gamma .
\end{aligned}
$$


Let us note that by ( [1], Theorem 15.2) the following estimate holds

$$
\begin{aligned}
\|w\|_{H^{2}(\Omega)} & \leq C_{9}\left(\left(\lambda_{k}(\alpha)+1\right)\|w\|_{L_{2}(\Omega)}\right. \\
& \left.+\left|\lambda_{k}(\tilde{\alpha})-\lambda_{k}(\alpha)\right|\left\|u_{k, \alpha}\right\|_{L_{2}(\Omega)}+|\tilde{\alpha}-\alpha|\left\|u_{k, \tilde{\alpha}}\right\|_{H^{1 / 2}(\Gamma)}\right)
\end{aligned}
$$

with the constant $C_{9}$ depends on $\Omega$. Thence, by the embedding theorem ( $[2]$, Theorem 5.1.7) we get

$$
\|u\|_{H^{1 / 2}(\Gamma)} \leq C_{10}\|u\|_{H^{1}(\Omega)}, \quad u \in H^{1}(\Omega)
$$

and with the inequalities (3.9), (5.2), (5.5) we obtain

$$
\|w\|_{H^{2}(\Omega)} \leq C_{11}|\tilde{\alpha}-\alpha|
$$

with the constant $C_{11}$ depends on $k$. Lemma 3 is proved.

Proof of Theorem 4. Let $m\left(\lambda_{k}^{D}\right)=1$ (the eigenvalue $\lambda_{k}^{D}$ is simple). By the estimates (3.13) and (4.3), applying Theorem 7 for $H=L_{2}(\Omega)$ with $A=A^{D}$, $B=A_{\alpha}$ we get

$$
\delta\left(u_{k}^{D}, u_{k, \alpha}\right) \leq \frac{2}{\varrho_{k}^{A^{D}}}\left\|A^{D}-A_{\alpha}\right\| \leq \frac{2 C_{3} C_{7}}{\varrho_{k}^{A^{D}} \alpha}, \quad \alpha>\alpha_{1} .
$$

Therefore, for normalized in $L_{2}(\Omega)$ eigenfunctions $u_{k}^{D}$ and $u_{k, \alpha}$ such that $\int_{\Omega} u_{k, \alpha} u_{k}^{D} d x \geq 0$ it follows from (5.7) and Lemma 1 that

$$
\left\|u_{k}^{D}-u_{k, \alpha}\right\|_{L_{2}(\Omega)} \leq \frac{2 \sqrt{2} C_{3} C_{7}}{\varrho_{k}^{A^{D}} \alpha}, \quad \alpha>\alpha_{1} .
$$

Now, we have the problems

$$
\begin{aligned}
& \Delta u_{k, \alpha}+\lambda_{k}(\alpha) u_{k, \alpha}=0 \quad \text { in } \Omega, \quad \frac{\partial u_{k, \alpha}}{\partial \nu}+\alpha u_{k, \alpha}=0 \quad \text { on } \Gamma, \\
& \Delta u_{k}^{D}+\lambda_{k}^{D} u_{k}^{D}=0 \quad \text { in } \Omega, \quad u_{k}^{D}=0 \quad \text { on } \Gamma .
\end{aligned}
$$

By $(5.9)-(5.10)$ the function $w=u_{k}^{D}-u_{k, \alpha}$ is a solution of the boundary value problem

$$
\begin{aligned}
-\Delta w+w & =\left(\lambda_{k}^{D}+1\right)\left(u_{k}^{D}-u_{k, \alpha}\right)+\left(\lambda_{k}^{D}-\lambda_{k}(\alpha)\right) u_{\alpha} & & \text { in } \Omega \\
w & =\frac{1}{\alpha} \frac{\partial u_{k, \alpha}}{\partial \nu} & & \text { on } \Gamma .
\end{aligned}
$$

Besides, the function

$$
\tilde{w}=w-\frac{1}{\alpha}\left(b, \nabla u_{k, \alpha}\right), \quad b(x)=\left(b_{1}(x), \ldots, b_{n}(x)\right) \in C^{2}(\bar{\Omega}),\left.\quad b\right|_{\Gamma}=\nu
$$

is a solution of the problem

$$
-\Delta \tilde{w}+\tilde{w}=h_{k, \alpha} \quad \text { in } \Omega, \quad \tilde{w}=0 \quad \text { on } \Gamma,
$$


where

$$
\begin{aligned}
h_{k, \alpha}= & \left(\lambda_{k}^{D}+1\right)\left(u_{k}^{D}-u_{k, \alpha}\right)+\left(\lambda_{k}^{D}-\lambda_{k}(\alpha)\right) u_{k, \alpha} \\
& +\frac{1}{\alpha}\left(\left(b, \nabla u_{k, \alpha}\right)-\Delta\left(b, \nabla u_{k, \alpha}\right)\right) \\
= & \left(\lambda_{k}^{D}+1\right)\left(u_{k}^{D}-u_{k, \alpha}\right)+\left(\lambda_{k}^{D}-\lambda_{k}(\alpha)\right) u_{\alpha} \\
& +\frac{1}{\alpha}\left(\left(b, \nabla u_{k, \alpha}\right)-\left(\Delta b, \nabla u_{k, \alpha}\right)-2 \sum_{i, j=1}^{n}\left(b_{j}\right)_{x_{i}}\left(u_{k, \alpha}\right)_{x_{j}}-\left(b, \nabla \Delta u_{k, \alpha}\right)\right) \\
= & \left(\lambda_{k}^{D}+1\right)\left(u_{k}^{D}-u_{k, \alpha}\right)+\left(\lambda_{k}^{D}-\lambda_{k}(\alpha)\right) u_{k, \alpha} \\
& +\frac{1}{\alpha}\left(\left(b-\Delta b, \nabla u_{k, \alpha}\right)-2 \sum_{i, j=1}^{n}\left(b_{j}\right)_{x_{i}}\left(u_{k, \alpha}\right)_{x_{i} x_{j}}+\lambda_{k}(\alpha)\left(b, \nabla u_{k, \alpha}\right)\right) \\
= & \left(\lambda_{k}^{D}+1\right)\left(u_{k}^{D}-u_{k, \alpha}\right)+\left(\lambda_{k}^{D}-\lambda_{k}(\alpha)\right) u_{k, \alpha} \\
& \left.+\frac{1}{\alpha}\left(\left(1+\lambda_{k}(\alpha)\right) b-\Delta b, \nabla u_{k, \alpha}\right)-2 \sum_{i, j=1}^{n}\left(b_{j}\right)_{x_{i}}\left(u_{k, \alpha}\right)_{x_{i} x_{j}}\right) .
\end{aligned}
$$

The eigenfunction $u_{k, \alpha}$ is a solution of the boundary problem

$$
\begin{array}{ll}
-\Delta u_{k, \alpha}+u_{k, \alpha}=\left(\lambda_{k}(\alpha)+1\right) u_{k, \alpha} & \text { in } \Omega, \\
\frac{\partial u_{k, \alpha}}{\partial \nu}+\alpha u_{k, \alpha}=0 & \text { on } \Gamma
\end{array}
$$

and by (3.4) satisfies the estimate

$$
\left\|u_{k, \alpha}\right\|_{H^{2}(\Omega)} \leq C_{3}\left(\lambda_{k}(\alpha)+1\right), \quad \alpha>\alpha_{1} .
$$

In domain with $C^{3}$ class boundary surface $u \in H^{3}(\Omega)$ ( [15], Chapter 4, Section 2, Theorem 4). Further we need an elliptic estimate to solution $u$ of the problem (3.2), (3.3):

$$
\|u\|_{H^{3}(\Omega)} \leq C_{12}\|h\|_{H^{1}(\Omega)}, \quad \alpha>0,
$$

with the constant $C_{12}$ is independent of $\alpha$. The inequality (5.13) is obtained by the same method as the estimate (3.4) in ( [9], P. 105-112). Let us give scheme of the proof. At first we denote:

$$
\left|\nabla^{p} u\right|^{2}=\sum_{j_{1}=1}^{n} \cdots \sum_{j_{p}=1}^{n} u_{x_{j_{1}} \ldots x_{j_{p}}}^{2} .
$$

For any function $u \in C^{4}(\Omega) \cap C^{3}(\bar{\Omega})$ consider the equality

$$
\left|\nabla^{3} u\right|^{2}=|\nabla \Delta u|^{2}+\operatorname{div}\left(\frac{1}{2} \nabla\left(\left|\nabla^{2} u\right|^{2}\right)-\sum_{i=1}^{n} \Delta u_{x_{i}} \nabla u_{x_{i}}\right),
$$

obtained by direct computation. Integrating the relation (5.14) on $\Omega$ and applying Gauss-Ostrogradskiy formula, we have the equality

$$
\int_{\Omega}\left|\nabla^{3} u\right|^{2} d x=\int_{\Omega}|\nabla \Delta u|^{2} d x+I,
$$


where

$$
I=\int_{\Gamma}\left(\frac{1}{2} \frac{\partial}{\partial \nu}\left(\left|\nabla^{2} u\right|^{2}\right)-\sum_{j=1}^{n} \Delta u_{x_{j}} \frac{\partial}{\partial \nu} u_{x_{j}}\right) d s .
$$

To obtain the equality $(5.15)$ for $u \in C^{3}(\bar{\Omega})$ it is sufficient to take a limit in (5.15) for the sequence of functions $u_{j} \in C^{4}(\Omega) \cap C^{3}(\bar{\Omega})$ such that $\lim _{j \rightarrow \infty} \| u-$ $u_{j} \|_{C^{3}(\bar{\Omega})}=0$. Now, let $u \in C^{3}(\bar{\Omega})$ be a solution of the problem (3.2), (3.3). By (3.2) we get

$$
\begin{aligned}
\int_{\Omega}\left|\nabla^{3} u\right|^{2} d x & =\int_{\Omega}|\nabla(u-h)|^{2} d x+I \\
& \leq 2\left(\int_{\Omega}|\nabla u|^{2} d x+\int_{\Omega}|\nabla h|^{2} d x\right)+I .
\end{aligned}
$$

Moreover, we have the energy estimate ( [9], Formula (35)):

$$
\int_{\Omega}\left(|\nabla u|^{2}+u^{2}\right) d x+\alpha \int_{\Gamma} u^{2} d s \leq \int_{\Omega} h^{2} d x, \quad \alpha>0
$$

and the following inequality ( [9], see Formulas (37), (39), (49), (54))

$$
\begin{aligned}
\int_{\Omega}\left|\nabla^{2} u\right|^{2} d x & +\alpha \int_{\Gamma}\left|\nabla_{\tau} u\right|^{2} d s \\
& \leq \int_{\Omega}(\Delta u)^{2} d x+(n-1) \int_{\Gamma} H(x)\left(\frac{\partial u}{\partial \nu}\right)^{2} d s \\
\alpha & >\max _{x \in \Gamma} \max _{1 \leq j \leq n-1}\left|k_{j}(x)\right|,
\end{aligned}
$$

where the vector $\nabla_{\tau} u=\nabla u-\frac{\partial u}{\partial \nu} \nu$ is a tangential gradient of the function $u$ on the surface $\Gamma, H(x)$ is a mean curvature and $k_{j}(x)$ are main curvatures of $\Gamma$ oriented by outward normal at the point $x$. To estimate the surface integral $I$ in (5.16) we will consider the element of integration in the local orthogonal coordinate system in the arbitrary point $x \in \Gamma$ such that $n$th axe direction coincides with $\nu$ and the origin is in $x$. Then, transforming $I$ by the technique of ( [13], Chapter 1, Section 7, Lemmas 1, 2', 3, Chapter 2, Section 3, Lemma 1'; [18]; [9]), with the estimates (5.17), (5.18) we obtain from (5.16) the inequality

$$
\int_{\Omega}\left|\nabla^{3} u\right|^{2} d x \leq C_{13} \int_{\Omega}\left(|\nabla h|^{2}+h^{2}\right) d x, \quad \alpha>0
$$

with the constant $C_{13}$ is independent of $\alpha$. Combine (3.4) and (5.19), we get the estimate (5.13) for solutions of the problem $(3.2)$, (3.3) from $C^{3}(\bar{\Omega})$. To prove the estimate (5.13) for solutions from $H^{3}(\Omega)$ we take a sequence of functions $u_{j} \in C^{3}(\bar{\Omega})$ satisfying (3.3) such that $\left\|u-u_{j}\right\|_{H^{3}(\Omega)} \rightarrow 0, j \rightarrow \infty$. Applying the estimate (5.19) to the functions $u_{j}$ we have

$$
\left\|u_{j}\right\|_{H^{3}(\Omega)} \leq C_{12}\left\|h_{j}\right\|_{H^{1}(\Omega)}, \quad \alpha \geq \alpha_{1},
$$

where $h_{j}=-\Delta u_{j}+u_{j}$. Therefore, $\lim _{j \rightarrow \infty}\left\|h_{j}\right\|_{H^{1}(\Omega)}=\|h\|_{H^{1}(\Omega)}$. Taking a limit in (5.20), we obtain the inequality (5.13) for solutions $u \in H^{3}(\Omega)$. 
Combining (1.7), (5.11) and (5.12), we obtain the estimate

$$
\begin{aligned}
\left\|h_{k, \alpha}\right\|_{L_{2}(\Omega)} \leq & \left(\lambda_{k}^{D}+1\right)\left\|u_{k}^{D}-u_{k, \alpha}\right\|_{L_{2}(\Omega)} \\
& +\left(\lambda_{k}^{D}-\lambda_{k}(\alpha)\right)\left\|u_{k, \alpha}\right\|_{L_{2}(\Omega)}+\frac{C_{14}}{\alpha}\left\|u_{k, \alpha}\right\|_{H^{2}(\Omega)} \\
\leq & C_{15}\left(\left\|u_{k}^{D}-u_{k, \alpha}\right\|_{L_{2}(\Omega)}+\frac{1}{\alpha}\right), \quad \alpha>\alpha_{1},
\end{aligned}
$$

where the constant $C_{15}$ is independent of $\alpha$, but may depends on $k$. It follows from the inequality ( $[15]$, Chapter 4, Section 2, Theorem 4) that

$$
\|\tilde{w}\|_{H^{2}(\Omega)} \leq C_{16}\left(\left\|u_{k}^{D}-u_{k, \alpha}\right\|_{L_{2}(\Omega)}+\frac{1}{\alpha}\right), \quad \alpha>\alpha_{1} .
$$

Combining (5.8), (5.13), (5.21) with (5.22), we get

$$
\begin{aligned}
\| u_{k}^{D} & -u_{k, \alpha}\left\|_{H^{2}(\Omega)}=\right\| \tilde{w}+\frac{1}{\alpha}\left(b, \nabla u_{k, \alpha}\right) \|_{H^{2}(\Omega)} \\
& \leq\|\tilde{w}\|_{H^{2}(\Omega)}+\frac{1}{\alpha}\left\|\left(b, \nabla u_{k, \alpha}\right)\right\|_{H^{2}(\Omega)} \\
& \leq C_{16}\left(\left\|u_{k}^{D}-u_{k, \alpha}\right\|_{L_{2}(\Omega)}+\frac{1}{\alpha}\right)+\frac{C_{17}}{\alpha}\left\|u_{k, \alpha}\right\|_{H^{3}(\Omega)} \\
& \leq C_{16}\left(\left\|u_{k}^{D}-u_{k, \alpha}\right\|_{L_{2}(\Omega)}+\frac{1}{\alpha}\right)+\left(\lambda_{k}^{D}+1\right) \frac{C_{12} C_{17}}{\alpha}\left\|u_{k, \alpha}\right\|_{H^{1}(\Omega)} \\
& \leq C_{16}\left(\left\|u_{k}^{D}-u_{k, \alpha}\right\|_{L_{2}(\Omega)}+\frac{1}{\alpha}\right)+\left(\lambda_{k}^{D}+1\right)^{3 / 2} \frac{C_{12} C_{17}}{\alpha} \\
& \leq C_{18}\left(\left\|u_{k}^{D}-u_{k, \alpha}\right\|_{L_{2}(\Omega)}+\frac{1}{\alpha}\right) \leq \frac{M_{k}}{\alpha}, \quad \alpha>\alpha_{1}
\end{aligned}
$$

with the constant $M_{k}$ independent on $\alpha$. Theorem 4 is proved.

\section{Derivative of eigenvalue and asymptotic expansion}

Proof of Theorem 2. For eigenfunctions $u_{k, \alpha}, u_{k, \alpha^{\prime}}$ we have the equalities

$$
\begin{aligned}
& \int_{\Omega}\left(\left(\nabla u_{k, \alpha^{\prime}}, \nabla u_{k, \alpha}\right)-\lambda_{k}\left(\alpha^{\prime}\right) u_{k, \alpha^{\prime}} u_{k, \alpha}\right) d x=-\alpha^{\prime} \int_{\Gamma} u_{k, \alpha^{\prime}} u_{k, \alpha} d s \\
& \int_{\Omega}\left(\left(\nabla u_{k, \alpha}, \nabla u_{k, \alpha^{\prime}}\right)-\lambda_{k}(\alpha) u_{k, \alpha} u_{k, \alpha^{\prime}}\right) d x=-\alpha \int_{\Gamma} u_{k, \alpha} u_{k, \alpha^{\prime}} d s .
\end{aligned}
$$

Therefore,

$$
\frac{\lambda_{k}\left(\alpha^{\prime}\right)-\lambda_{k}(\alpha)}{\alpha^{\prime}-\alpha}=\frac{\int_{\Gamma} u_{k, \alpha^{\prime}} u_{k, \alpha} d s}{\int_{\Omega} u_{k, \alpha^{\prime}} u_{k, \alpha} d x} .
$$

Now, suppose that $\int_{\Omega} u_{k, \alpha} u_{k, \alpha^{\prime}} d x \geq 0$. By Lemma 3 and inequality (5.6) we obtain from (6.1) that

$$
\lambda_{k}^{\prime}(\alpha)=\lim _{\alpha^{\prime} \rightarrow \alpha} \frac{\int_{\Gamma} u_{k, \alpha^{\prime}} u_{k, \alpha} d s}{\int_{\Omega} u_{k, \alpha^{\prime}} u_{k, \alpha} d x}=\frac{\int_{\Gamma} u_{k, \alpha}^{2} d s}{\int_{\Omega} u_{k, \alpha}^{2} d x} .
$$


Theorem 2 is proved.

Proof of Theorem 3. Let $m\left(\lambda_{k}^{D}\right)=1$. We suppose $\int_{\Omega} u_{k, \alpha} u_{k}^{D} d x \geq 0$. For the normalized eigenfunction $u_{k}^{D}$ the relation (2.2) is equivalent to

$$
\lim _{\alpha \rightarrow+\infty} \frac{\lambda_{k}(\alpha)-\lambda_{k}^{D}}{1 / \alpha}=-\int_{\Gamma}\left(\frac{\partial u_{k}^{D}}{\partial \nu}\right)^{2} d s .
$$

Let us note that the numerator $\lambda_{k}(\alpha)-\lambda_{k}^{D}$ in the fraction in (6.2) by (1.7) tends to zero at $\alpha \rightarrow+\infty$. By the formula (2.1) and the boundary condition (1.2) we have

$$
\lambda_{k}^{\prime}(\alpha)=\int_{\Gamma} u_{k, \alpha}^{2} d s=\frac{1}{\alpha^{2}} \int_{\Gamma}\left(\frac{\partial u_{k, \alpha}}{\partial \nu}\right)^{2} d s
$$

Therefore,

$$
\lim _{\alpha \rightarrow+\infty} \frac{\lambda_{k}^{\prime}(\alpha)}{-1 / \alpha^{2}}=-\lim _{\alpha \rightarrow+\infty} \int_{\Gamma}\left(\frac{\partial u_{k, \alpha}}{\partial \nu}\right)^{2} d s .
$$

Let us prove that

$$
\lim _{\alpha \rightarrow+\infty} \int_{\Gamma}\left(\frac{\partial u_{k, \alpha}}{\partial \nu}\right)^{2} d s=\int_{\Gamma}\left(\frac{\partial u_{k}^{D}}{\partial \nu}\right)^{2} d s .
$$

By the inequality (5.23) and the embedding theorem ( [2], Theorem 5.1.7) we have

$$
\begin{aligned}
& \int_{\Gamma}\left(\frac{\partial u_{k}^{D}}{\partial \nu}-\frac{\partial u_{k, \alpha}}{\partial \nu}\right)^{2} d s \leq \int_{\Gamma}\left|\nabla\left(u_{k}^{D}-u_{k, \alpha}\right)\right|^{2} d s \\
& \quad \leq C_{19}\left(\left\|\nabla^{2}\left(u_{k}^{D}-u_{k, \alpha}\right)\right\|_{L_{2}(\Omega)}^{2}+\left\|\nabla\left(u_{k}^{D}-u_{k, \alpha}\right)\right\|_{L_{2}(\Omega)}^{2}\right) \\
& \quad \leq C_{19}\left\|u_{k}^{D}-u_{k, \alpha}\right\|_{H^{2}(\Omega)} \leq C_{19} M_{k} / \alpha \rightarrow 0, \quad \alpha \rightarrow+\infty .
\end{aligned}
$$

Using (6.5), we obtain the relation (6.4). Now, by L'Hôpital-Bernoulli theorem the equality (6.2) follows from (6.3). Proof of the Theorem 3 is completed.

\section{References}

[1] S. Agmon, A. Douglis and L. Nirenberg. Estimates near the boundary for solutions of elliptic partial differential equations satisfying general boundary conditions. I. Communications on Pure Applied Mathematics, 12(4):623-727, 1959. https://doi.org/10.1002/cpa.3160120405.

[2] M. Agranovich. Sobolev Spaces, Their Generalizations and Elliptic Problems in Smooth and Lipschitz Domains. Springer Monographs in Mathematics. Springer International Publishing, Berlin, 2015. https://doi.org/10.1007/9783-319-14648-5.

[3] R. Courant and D. Hilbert. Methods of Mathematical Physics, volume I. Wiley, New York, 1989.

[4] D. Daners and J.B. Kennedy. On the asymptotic behaviour of the eigenvalues of a Robin problem. Differential and Integral Equations, 23(7-8):659-669, 2010. 
[5] P. Exner, A.Minakov and L. Parnovski. Asymptotic eigenvalue estimates for a Robin problem with a large parameter. Portugaliae Mathematica, 72(2):141-156, 2014. https://doi.org/10.4171/PM/1945.

[6] A.V. Filinovskiy. Asymptotic behavior of the first eigenvalue of the Robin problem. On the seminar on qualitative theory of differential equations at Moscow State University. Differential Equations, 47(11):1680-1696, 2011. https://doi.org/10.1134/S0012266111110152.

[7] A.V. Filinovskiy. Estimates of eigenvalues of a boundary value problem with a parameter. Mathematical Communications, 19(3):531-543, 2014.

[8] A.V. Filinovskiy. On the eigenvalues of a Robin problem with a large parameter. Mathematica Bohemica, 139(2):341-352, 2014.

[9] A.V. Filinovskiy. On the estimates of eigenvalues of the boundary value problem with large parameter. Tatra Mountains Mathematical Publications, 63(1):101113, 2015. https://doi.org/10.1515/tmmp-2015-0023.

[10] J.L. Kazdan. Perturbation of complete orthonormal sets and eigenfunction expansions. Proceedings of the American Mathematical Society, 27(3):506-510, 1971.

[11] V.A. Kondratiev and E.M. Landis. Qualitative theory of second order linear partial differential equations. In Partial Differential Equations - 3, volume 32 of Itogi Nauki i Tekhniki. Sovremennye Problemy Matematiki. Fundamental'nye Napravleniya., pp. 99-215, Moscow, 1988. VINITI.

[12] A.A. Lacey, J.Sabina and J.R. Ockendon. Multidimensional reaction diffusion equations with nonlinear boundary conditions. SIAM Journal on Applied Mathematics, 58(5):1622-1647, 1998. https://doi.org/10.1137/S0036139996308121.

[13] O.A. Ladyzhenskaya. The mixed Problem for a Hyperbolic Equation. GTTI, Moscow, 1953.

[14] Y. Lou and M. Zhu. A singularly perturbed linear eigenvalue problem in $C^{1}$ domains. Pacific Journal of Mathematics, 214(2):323-334, 2004. https://doi.org/10.2140/pjm.2004.214.323.

[15] V.P. Mikhailov. Partial Differential Equations. Nauka, Moscow, 1983.

[16] S.G. Mikhlin. Numerical Realization of Variational Methods. Nauka, Moscow, 1966 .

[17] K. Pankrashkin and N. Popoff. Mean curvature bounds and eigenvalues of Robin Laplacians. Calculus of Variations and Partial Differential Equations, 54(2):1947-1961, 2015. https://doi.org/10.1007/s00526-015-0850-1.

[18] H.L. Smolitskiy. Some integral estimates to the derivatives of solutions of the wave equation. Doklady Akademii Nauk, 73(2):279-282, 1950.

[19] R. Sperb. Untere und obere Schranken für den tiefsten Eigenwert elastisch gestützten Membran. Zeitschrift fr angewandte Mathematik und Physik ZAMP, 23(2):231-244, 1972. https://doi.org/10.1007/BF01593087. 